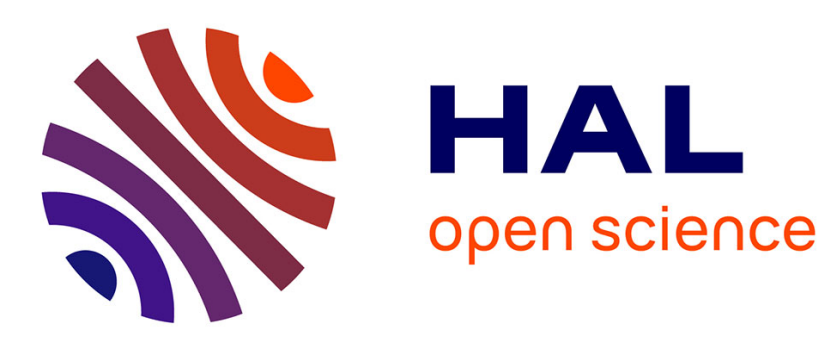

\title{
On the restricted cores and the bounded core of games on distributive lattices
}

Michel Grabisch, Peter Sudhölter

\section{To cite this version:}

Michel Grabisch, Peter Sudhölter. On the restricted cores and the bounded core of games on distributive lattices. 2012. halshs-00748331

\section{HAL Id: halshs-00748331 \\ https://shs.hal.science/halshs-00748331}

Submitted on 5 Nov 2012

HAL is a multi-disciplinary open access archive for the deposit and dissemination of scientific research documents, whether they are published or not. The documents may come from teaching and research institutions in France or abroad, or from public or private research centers.
L'archive ouverte pluridisciplinaire HAL, est destinée au dépôt et à la diffusion de documents scientifiques de niveau recherche, publiés ou non, émanant des établissements d'enseignement et de recherche français ou étrangers, des laboratoires publics ou privés. 


\section{Documents de Travail du Centre d'Economie de la Sorbonne}
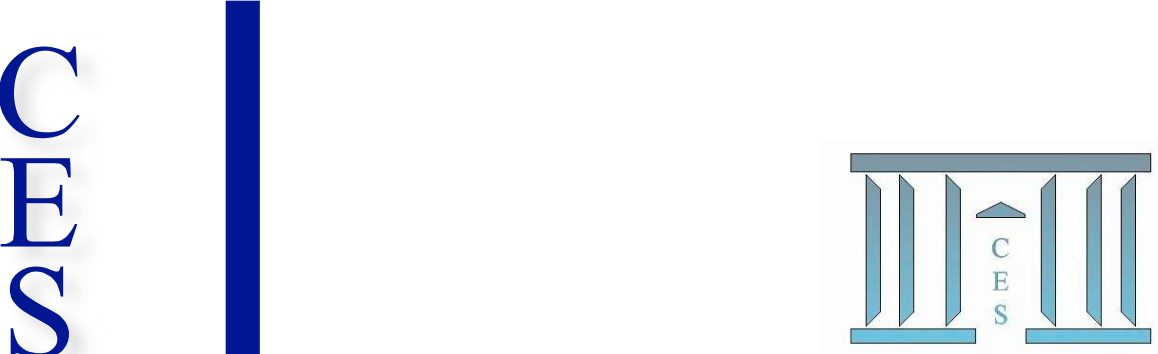

On the restricted cores and the bounded core of games on distributive lattices

Michel GRABISCH, Peter SUDHÖLTER

2012.67

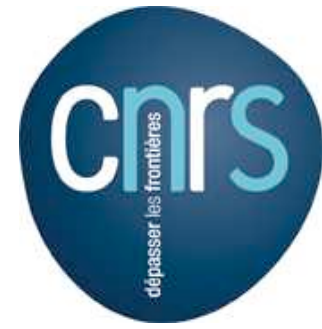




\title{
On the restricted cores and the bounded core of games on distributive lattices
}

\author{
Michel GRABISCH* ${ }^{*} \quad$ Peter SUDHÖLTER ${ }^{\dagger}$
}

\begin{abstract}
We consider TU-games with restricted cooperation, where the set of feasible coalitions is a distributive lattice, hence generated by a partial order on the set of players. In such a situation, the core may be unbounded, and one has to select a bounded part of the core as a solution concept. The restricted core is obtained by imposing equality constraints in the core for sets belonging to so-called normal collections, resulting (if nonempty) in the selection of a bounded face of the core. The bounded core proves to be the union of all bounded faces (restricted cores). The paper aims at investigating in depth the relation between the bounded core and restricted cores, as well as the properties and structures of the restricted cores and normal collections. In particular, it is found that a game is convex if and only if all restricted cores corresponding to the minimal nested normal collections are nonempty. Moreover, in this case the union of these restricted cores already covers the bounded core.
\end{abstract}

Keywords: TU-game, restricted cooperation, distributive lattice, core, extremal rays, faces of the core

JEL Classification: C71

MSC Code: 91A12, 06D99

\section{Introduction}

In cooperative game theory, for a given set of players $N$, TU-games are functions $v: 2^{N} \rightarrow$ $\mathbb{R}$ which express for each nonempty coalition $S \subseteq N$ of players the best they can achieve by cooperation. In the classical setting, every coalition may form without any restriction, i.e., the domain of $v$ is indeed $2^{N}$. In practice, this assumption is often unrealistic, since some coalitions may not be feasible for various reasons, e.g., players are political parties with divergent opinions, or have restricted communication abilities, or a hierarchy exists among players, and the formation of coalitions must respect the hierarchy, etc.

\footnotetext{
*Corresponding author. Paris School of Economics, University of Paris I. 106-112, Bd. de l'Hôpital, 75013 Paris, France. Tel (+33)14407-8285, Fax (+33)14407-8301. Email: michel.grabisch@univ-paris1.fr

$\dagger$ Department of Business and Economics and COHERE, University of Southern Denmark. Campusvej 55, 5230 Odense M, Denmark. Email: psu@sam.sdu.dk
} 
Many studies have been done on games defined on specific subdomains of $2^{N}$, e.g., antimatroids (Algaba et al., 2004), convex geometries (Bilbao and Edelman, 2000), distributive lattices (Faigle and Kern, 1992), or others (Bilbao et al., 2000). In this paper, we focus on the case of distributive lattices. To this end, we assume that there exists some partial order $\preceq$ on $N$ describing some hierarchy or precedence constraint among players, as in Faigle and Kern (1992). We say that a coalition $S$ is feasible if the coalition contains all its subordinates, i.e., $i \in S$ implies that any $j \preceq i$ belongs to $S$ as well. Then by Birkhoff's theorem, feasible coalitions form a distributive lattice.

The main problem in cooperative game theory is to define a rational solution of the game, that is, supposing that the grand coalition $N$ will form, how to share among its members the total worth $v(N)$. The core is the most popular solution concept, since it ensures stability of the game, in the sense that no coalition has an incentive to deviate from the grand coalition. For classical TU-games, the core is either empty or a convex bounded polyhedron. However, for games whose cooperation is restricted, the study of the core is much more complex, since it may be unbounded or even contain no vertices (see a survey in Grabisch (2009)). For the case of games with precedence constraints, it is known that the core is always unbounded or empty, but contains no line (i.e., it has vertices). The problem arises then, to select a significant bounded part of the core as a reasonable concept of solution, since unbounded payments make no sense.

As far as we know, two remedies have been proposed in the literature for this problem. The first one is to consider restricted cores, i.e., to impose in the core further equality constraints $x(S)=v(S)$ for all $S$ in some collection $\mathcal{N}$, which can be interpreted as binding constraints for some coalitions, in order to exclude any extremal ray in the core (Grabisch and Xie, 2011; Grabisch, 2011). Such a collection is called a normal collection. The second one was proposed by the authors, by considering core elements such that every player takes the maximum of her direct subordinates, in the sense that any transfer from a subordinate to her boss would result in a payoff vector outside the core. The resulting set is called the bounded core (Grabisch and Sudhölter, to appear). It happens that the two concepts are related as follows: the bounded core is the union of all restricted cores, taken over all possible normal collections.

The aim of this paper is to investigate more deeply the relation between the two concepts, and to complete the results presented in Grabisch (2011) for restricted cores. In particular, we introduce several remarkable normal collections and study their properties. Nested normal collections, i.e., collections whose sets form a chain, play a fundamental rôle, in particular for convex games. We show that for convex games, only minimal (in the size of the collection) nested normal collections matter, in the sense that the bounded core is the union of restricted cores corresponding to minimal nested collections. Moreover, such restricted cores are never empty. If the game is strictly convex, then this union does not contain any redundant term. Finally, we show that minimal nested collections can be generated by a special class of linear extensions of the partial order $\preceq$ on $N$. Besides, we show a generalization of the well-known Shapley-Ichiishi theorem for games with precedence constraints.

The paper is organized as follows. Section 2 establishes the basic material for the rest of the paper, and presents the notions of restricted core, normal collection and bounded core. Section 3 studies the set of normal collections, introduces properties and remarkable 
collections. It shows also how nested collections can be obtained by a closure operator on a certain class of normal collections. Section 4 studies under which conditions the restricted core is nonempty, and provides a balancedness condition. Section 5 investigates in depth the case of convex games, showing the fundamental rôle played by minimal nested normal collections.

\section{Notation, definitions and preliminaries}

The characteristic function of a set $S$ is denoted by $1^{S}$. We often omit braces for singletons, writing, e.g., $1^{i}$ instead of $1^{\{i\}}$.

Let $(P, \preceq)$ be a finite partially ordered set (poset for short), that is, a finite set $P$ endowed with a reflexive, antisymmetric and transitive relation (see, e.g., Davey and Priestley (1990)). We denote by $\prec$ the asymmetric part of $\preceq$. We say that $x \in P$ covers $y \in P$, and we denote it by $y \prec x$, if $y \prec x$ and there is no $z \in P$ such that $y \prec z \prec x$.

We denote by $\min (P)$ and $\max (P)$ respectively the set of the minimal and maximal elements of $(P, \preceq)$. The dual of the poset $(P, \preceq)$, denoted by $\left(P, \preceq^{\partial}\right)$ (or simply $\left.P^{\partial}\right)$, is the set $P$ endowed with the reverse order, i.e., $x \preceq y$ if and only if $y \preceq^{\partial} x$.

Throughout the paper, it is understood that any subset $Q$ of a poset $(P, \preceq)$ is endowed with $\preceq$ restricted to $Q$ (we do not use a special symbol for the restriction).

A chain $C \subseteq P$ is a subset of $P$ such that for any two elements $x, y \in C$, we have $x \preceq y$ or $y \preceq x$. The chain is maximal if no other chain contains it, or equivalently if $C=\left\{x_{1}, \ldots, x_{q}\right\}$, with $x_{1} \prec \cdot x_{2} \prec \cdot \ldots \prec \cdot x_{q}$ and $x_{1} \in \min (P), x_{q} \in \max (P)$. Its length is $q-1$. The height of $i \in P$, denoted by $h(i)$, is the length of a longest chain from a minimal element to $i$. Elements of same height $k$ form level $k$, denoted by $L_{k}$. Hence, $L_{0}=\min (P)$ is the set of all minimal elements, $L_{1}=\min \left(P \backslash L_{0}\right), L_{2}=\min \left(P \backslash\left(L_{0} \cup L_{1}\right)\right)$, etc. The height of $N$, denoted by $h(N)$, is the maximum of $h(i)$ taken over all elements of $N$. Similarly, we define the depth $d(i)$ of an element $i \in N$ as its height in the dual poset $P^{\partial}$. We denote by $D_{0}$ the set of all elements of depth 0 and we have $D_{0}=\max (P)$, $D_{1}=\max \left(P \backslash D_{0}\right), D_{2}=\max \left(P \backslash\left(D_{0} \cup D_{1}\right)\right)$, etc.

A lattice is a poset $(L, \preceq)$ where for each $x, y \in L$, their supremum $x \vee y$ and infimum $x \wedge y$ exist. The lattice is distributive if $\vee, \wedge$ obey distributivity.

A subset $Q \subseteq P$ is a downset of $P$ if $x \in Q$ and $y \preceq x$ implies $y \in Q$. We denote by $\mathcal{O}(P)$ the set of downsets of $(P, \preceq)$. It is a well-known fact that $(\mathcal{O}(P), \subseteq)$ is a distributive lattice and every distributive lattice arises that way (Birkhoff, 1933). We denote by $\downarrow x$ the downset generated by an element $x \in P$, that is, $\downarrow x=\{y \in P \mid y \preceq x\}$. Similarly, for any $Q \subseteq P, \downarrow Q=\bigcup_{x \in Q} \downarrow x$.

Let $N$ be a finite set of $n$ players. A set system $\mathcal{F}$ on $N$ is a collection of subsets of $N$ containing $N$ and $\emptyset$. Any nonempty subset in $\mathcal{F}$ is called a feasible coalition. We define a cooperative $T U$-game with restricted cooperation (or simply a game) on $\mathcal{F}$ as the pair $(\mathcal{F}, v)$, with $v: \mathcal{F} \rightarrow \mathbb{R}$, such that $v(\emptyset)=0$.

In this paper we focus on a particular case of set systems, introduced by Faigle and Kern (1992) (games with precedence constraints). We consider a partial order $\preceq$ on $N$, which may express precedence constraints among players, or hierarchical relations. A coalition $S$ is feasible if whenever $i \in S$, all subordinates of $i$ also belong to $S$, i.e., $S$ is 
a downset of $(N, \preceq)$. In other words, $\mathcal{F}=\mathcal{O}(N)$, hence $\mathcal{F}$ is a distributive lattice where supremum and infimum are respectively $\cup, \cap$.

A game $(\mathcal{F}, v)$ with $\mathcal{F}=\mathcal{O}(N)$ is convex if

$$
v(S \cup T)+v(S \cap T) \geqslant v(S)+v(T)
$$

for every $S, T \in \mathcal{F}$. It is superadditive if the above inequality is restricted to disjoint sets $S, T$. It is strictly convex if the above inequality is strict for all $S, T \in \mathcal{F}$ such that $S \backslash T \neq \emptyset$ and $T \backslash S \neq \emptyset$.

The following lemma extends a classical result when $\mathcal{F}=2^{N}$.

Lemma 1. A game $(\mathcal{F}, v)$ is convex if and only if for all $i \in N$, for all $P \subseteq Q \subseteq N \backslash i$ such that $P \cup i, Q \in \mathcal{F}$,

$$
v(P \cup i)-v(P) \leqslant v(Q \cup i)-v(Q) .
$$

Proof. In order to show that (1) implies (2), put $S=P \cup i$ and $T=Q$, and observe that $S \cap T=P$ and $S \cup T=Q \cup i$. For the other implication we may assume that $S \backslash T \neq \emptyset$ so that there exists $i_{1}, \ldots, i_{p} \in N$, where $p=|S \backslash T|$, such that $(S \cap T) \cup\left\{i_{1}, \ldots, i_{m}\right\} \in \mathcal{F}$ for all $m=1, \ldots, p-1$ and $S \backslash T=\left\{i_{1}, \ldots, i_{p}\right\}$ (it suffices that $i_{k} \nprec i_{\ell}$ for $k>\ell$ ). By $(2)$,

$$
\begin{aligned}
v(S)-v(S \cap T) & =\sum_{m=1}^{p}\left(v\left((S \cap T) \cup\left\{i_{1}, \ldots, i_{m}\right\}\right)-v\left((S \cap T) \cup\left\{i_{1}, \ldots, i_{m}\right\}\right)\right) \\
& \leqslant \sum_{m=1}^{p}\left(v\left(T \cup\left\{i_{1}, \ldots, i_{m}\right\}\right)-v\left(T \cup\left\{i_{1}, \ldots, i_{m-1}\right\}\right)\right)=v(S \cup T)-v(T) .
\end{aligned}
$$

The core of a game $(\mathcal{F}, v)$ is defined as follows:

$$
\mathcal{C}(\mathcal{F}, v)=\left\{x \in \mathbb{R}^{n} \mid x(S) \geqslant v(S) \text { for all } S \in \mathcal{F}, \text { and } x(N)=v(N)\right\},
$$

where $x(S)=\sum_{i \in S} x_{i}$, with the convention $x(\emptyset)=0$. If no ambiguity menaces, we write simply $\mathcal{C}(v)$. By definition, it is a convex closed polyhedron. Unless $\mathcal{F}=2^{N}$, the core is unbounded or empty. Derks and Gilles (1995) showed (as well as Tomizawa (1983) in a refined form) that it contains no line, and found its rays (see also Fujishige (2005, Theorem 3.26)). It is well known from the theory of polyhedra that the core can be written as the Minkowski sum of its convex part and its conic part:

$$
\mathcal{C}(v)=\operatorname{conv}(\operatorname{ext}(\mathcal{C}(v)))+\mathcal{C}(0)
$$

where $\operatorname{ext}(\cdot), \operatorname{conv}(\cdot)$ denote the extreme points of some convex set, and the convex hull of a set. Note that the conic part is obtained by replacing $v$ by the zero function, hence the conic part depends solely on $\mathcal{F}$. Extremal rays of the core are generated by $1^{j}-1^{i}$ for every $i, j \in N$ such that $j \prec \cdot i$ in $(N, \preceq)$. Therefore, extremal rays correspond bijectively to edges in the Hasse diagram of $(N, \preceq)$. 
To avoid unboundedness, a natural solution is to take a certain subset of the core. In Grabisch (2011), some of the inequalities $x(S) \geqslant v(S)$ are turned into equalities so that no extremal ray exists any more. These equalities can be considered as additional binding constraints on certain coalitions. We call normal collection any collection $\mathcal{N} \subseteq \mathcal{F} \backslash\{\emptyset, N\}$ such that

$\mathcal{C}_{\mathcal{N}}(\mathcal{F}, v)=\left\{x \in \mathbb{R}^{n} \mid x(S) \geqslant v(S) \forall S \in \mathcal{F}, \quad x(S)=v(S) \forall S \in \mathcal{N}\right.$, and $\left.x(N)=v(N)\right\}$

is bounded for all games $(\mathcal{F}, v)$ on $\mathcal{F}$. Note that $\mathcal{N}$ is normal if and only if $\mathcal{C}_{\mathcal{N}}(0)=\{0\}$. It is remarked that the empty collection is normal if and only if $\mathcal{F}=2^{N}$. We call $\mathcal{C}_{\mathcal{N}}(\mathcal{F}, v)$ the restricted core w.r.t. $\mathcal{N}$, and we write simply $\mathcal{C}_{\mathcal{N}}(v)$ if no ambiguity menaces.

We denote by $\mathcal{N C}(\mathcal{F})$ the set of normal collections on $\mathcal{F}$. In Grabisch (2011), several normal collections are proposed (see Section 3.1). It is proved that a normal collection contains at least $h(N)$ sets, where $h(N)$ is the height of $(N, \preceq)$.

We say that an extremal ray $r$ of $\mathcal{C}(0)$ is deleted by equality $x(S)=0$ if $\mathcal{C}_{\{S\}}(0)=$ $\{x \in \mathcal{C}(0) \mid x(S)=0\}$ does not contain $r$ any more. The following result from (Grabisch, 2011) is fundamental.

Lemma 2. Let $\mathcal{F}=\mathcal{O}(N)$. For $i, j \in N$ such that $j \prec \cdot i$, the extremal ray generated by $1^{j}-1^{i}$ is deleted by equality $x(S)=0$ if and only if $S \ni j$ and $S \not \supset i$.

Geometrically, a restricted core $\mathcal{C}_{\mathcal{N}}(v)$, whenever nonempty, is a bounded face of the core $\mathcal{C}(v)$ because it is bounded and defined by just turning some constraints that determine the core into binding constraints.

On the other hand, the authors have proposed the notion of bounded core (Grabisch and Sudhölter, to appear). The bounded core $\mathcal{C}^{b}(v)$ of $(\mathcal{F}, v)$ is the set of elements of $\mathcal{C}(v)$ that satisfy the following condition for any $i, j \in N$ such that $j \prec \cdot i$ : There is no $\epsilon>0$ such that $x+\epsilon 1^{i}-1^{j} \in \mathcal{C}(v)$. Hence the bounded core is the set of core elements such that every player takes the maximum of her direct subordinates, in the sense that any transfer from a subordinate to her boss would result in a payoff vector outside the core. Also, the bounded core is the union of all bounded faces of $\mathcal{C}(v)$. If nonempty, it coincides with the core if and only if $\mathcal{F}=2^{N}$.

The following result shows the relation between the two concepts (Grabisch and Sudhölter, to appear).

Proposition 1. Let $\mathcal{F}=\mathcal{O}(N)$, and consider any game $(\mathcal{F}, v)$. Then

$$
\mathcal{C}^{b}(v)=\bigcup_{\mathcal{N} \in \mathcal{N} \mathcal{C}(\mathcal{F})} \mathcal{C}_{\mathcal{N}}(v)
$$

\section{The set of normal collections}

Let $(N, \preceq)$ be a poset and consider $\mathcal{F}=\mathcal{O}(N)$. In order to avoid pathologic cases we assume throughout this section that $\preceq \neq \emptyset$, i.e., $h(N)>0$. We now define some possible properties of a normal collection.

Definition 1. Let $\mathcal{N}=\left\{N_{1}, \ldots, N_{q}\right\}$ be a normal collection. 
(i) $\mathcal{N}$ is a minimal collection if no proper subcollection is normal.

(ii) $\mathcal{N}$ is a thin collection if no $S \in \mathcal{N}$ may be replaced by a proper subset of $S$ without losing normality.

(iii) $\mathcal{N}$ is a short collection if it contains exactly $h(N)$ subsets.

(iv) $\mathcal{N}$ is a nested collection if it is a chain in $\mathcal{F}$.

Note that any short normal collection is minimal, but the converse is not true (see Example 4).

We give some elementary properties of normal collections.

Lemma 3. Let $\mathcal{N}=\left\{N_{1}, \ldots, N_{q}\right\}$ be a normal nested collection. Then $N_{k} \backslash N_{k-1}$ is an antichain in $(N, \preceq)$ for $k=1, \ldots, q$, with $N_{0}=\emptyset$.

Proof. If $N_{k} \backslash N_{k-1}$ is not an antichain, then there exist $i, j \in N_{k} \backslash N_{k-1}$ such that $i \prec j$. Since $\mathcal{N}$ is nested, no set in $\mathcal{N}$ will contain $i$ and not $j$. Then by Lemma 2 , the ray $1^{i}-1^{j}$ is not deleted by $\mathcal{N}$.

Lemma 4. Suppose that $\mathcal{N}$ is a collection containing a set $S$ such that $N \backslash S \in \mathcal{F}$. If $\mathcal{N}$ is normal, then $\mathcal{N} \backslash\{S\}$ is normal.

Proof. If there were an element $S \in \mathcal{N}$ such that $N \backslash S \in \mathcal{F}$, then the condition $x(S)=0$ would not eliminate any extremal ray because $j \in S$ and $j \prec \cdot i$ would imply $i \in S$. Therefore, $S$ can be discarded from $\mathcal{N}$.

Lemma 5. Let $\mathcal{N}$ be a normal collection that contains two disjoint sets $P$ and $Q$ with $P \cup Q \neq N$. If $\mathcal{N}^{\prime}=(\mathcal{N} \backslash\{P, Q\}) \cup\{P \cup Q\}$, then $\mathcal{N}^{\prime}$ is a normal collection, and $\mathcal{C}_{\mathcal{N}}(\mathcal{F}, v) \subseteq \mathcal{C}_{\mathcal{N}^{\prime}}(\mathcal{F}, v)$ for every superadditive game $(\mathcal{F}, v)$.

Proof. As $\mathcal{F}=\mathcal{O}(N), P \cup Q \in \mathcal{F}$. Let $i, j \in N$ such that $j \prec \cdot i$. If $j \in P \not \supset i$, then $i \notin Q$ because $Q \in \mathcal{O}(N)$ and $P \cap Q=\emptyset$. Similarly, if $j \in Q \not \supset i$, then $i \notin P$. Hence $\mathcal{N}^{\prime}$ is still normal by Lemma 2. Now, let $x \in \mathcal{C}_{\mathcal{N}}(v)$. Then $x(T)=v(T)$ for all $T \in \mathcal{N}^{\prime} \backslash\{P \cup Q\}, x(S) \geqslant v(S)$ for all $S \in \mathcal{F} \backslash \mathcal{N}^{\prime}, x(N)=v(N), x(P)=v(P)$, and $x(Q)=v(Q), x(P \cup Q) \geqslant v(P \cup Q) \geqslant v(P)+v(Q)$, where the last inequality is valid by superadditivity. Hence $x(P \cup Q)=v(P \cup Q)$, so that $x \in \mathcal{C}_{\mathcal{N}^{\prime}}(v)$.

\subsection{Some remarkable normal collections}

We introduce some notation. We say that an element $x$ of a poset $(P, \preceq)$ is disconnected if it is incomparable with any other element of $P$ (i.e., it has no link in the Hasse diagram of $(P, \preceq)$ ), equivalently, if it is both minimal and maximal. We denote by $P^{\circ}$ the set of elements of $P$ which are not disconnected:

$$
P^{\circ}=P \backslash(\min (P) \cap \max (P)) .
$$

For any subset $Q \subseteq P$, we define $\underline{Q}=\{y \in P \mid y \prec \cdot x$ for some $x \in Q\}$. 
We introduce the upwards normal collection $\mathcal{N}^{\mathrm{u}}=\left\{N_{1}^{\mathrm{u}}, \ldots, N_{q}^{\mathrm{u}}\right\}$, defined as follows (Grabisch, 2011).

$$
\begin{aligned}
N_{1}^{\mathrm{u}} & =\min \left(N^{\circ}\right) \\
N_{2}^{\mathrm{u}} & =\downarrow \min \left(\left(N \backslash N_{1}^{\mathrm{u}}\right)^{\circ}\right)=\downarrow \min \left(\left(N \backslash L_{0}\right)^{\circ}\right) \\
\vdots & =\vdots \\
N_{q}^{\mathrm{u}} & =\downarrow \min \left(\left(N \backslash\left(N_{1}^{\mathrm{u}} \cup \cdots \cup N_{q-1}^{\mathrm{u}}\right)\right)^{\circ}\right)=\downarrow \min \left(\left(N \backslash\left(L_{0} \cup \cdots \cup L_{q-2}\right)\right)^{\circ}\right)
\end{aligned}
$$

where $q$ is minimal such that $N \backslash\left(N_{1}^{\mathrm{u}} \cup \cdots \cup N_{q}^{\mathrm{u}}\right)^{\circ}=\emptyset$ (for the definitions of $h(P), L_{k}, D_{k}$, etc., see Section 2). Note that since $\left(N \backslash\left(N_{1}^{\mathrm{u}} \cup \cdots \cup N_{k-1}^{\mathrm{u}}\right)\right)^{\circ}=\left(N \backslash\left(L_{0} \cup \cdots \cup L_{k-2}\right)\right)^{\circ}$ for $k>1$, we deduce that $q=h(N)$, hence the upwards collection is short, therefore minimal. Grabisch (2011) proved that the upwards collection is also thin.

The upwards collection is built from the successive removal of minimal elements, hence its name. One can proceed as well from maximal elements. This gives the downwards collection $\mathcal{N}^{\mathrm{d}}=\left\{N_{1}^{\mathrm{d}}, \ldots, N_{q}^{\mathrm{d}}\right\}$, defined as follows.

$$
\begin{aligned}
N_{1}^{\mathrm{d}} & =\lfloor\underline{\max (N)} \\
N_{2}^{\mathrm{d}} & =\underline{\lfloor\max (N \backslash \max (N))}=\underline{\left\lfloor\max \left(N \backslash D_{0}\right)\right.} \\
\vdots & =\vdots \\
N_{q}^{\mathrm{d}} & =\underline{\left\lfloor\max \left(N \backslash\left(D_{0} \cup \cdots \cup D_{q-2}\right)\right)\right.}
\end{aligned}
$$

with $q=h(N)$ again, since $\max \left(N \backslash\left(D_{0} \cup \cdots \cup D_{h(N)-1}\right)\right)=\emptyset$. Therefore the downwards collection is short, hence minimal. Observe that the operation ()$^{\circ}$ is not necessary here, since any disconnected element is eliminated by the () operation.

The downwards collection can be expressed in a simpler way. Observe that $\underline{N}=$ $D_{1} \cup \cdots \cup D_{h(N)}, N \backslash D_{0}=D_{2} \cup \cdots \cup D_{h(N)}$, etc. Moreover, $\underline{N}=\downarrow \max (N)$, $N \backslash D_{0}=\downarrow \max \left(N \backslash \overline{\left.D_{0}\right)}\right.$, etc., hence we obtain

$$
\begin{aligned}
N_{1}^{\mathrm{d}} & =D_{1} \cup D_{2} \cup \cdots \cup D_{h(N)} \\
N_{2}^{\mathrm{d}} & =D_{2} \cup \cdots \cup D_{h(N)} \\
\vdots & =\vdots \\
N_{h(N)}^{\mathrm{d}} & =D_{h(N)},
\end{aligned}
$$

which shows that the downwards collection is nested. In addition, we have the following result.

Proposition 2. The downwards collection is thin.

Proof. Consider $N_{\ell}^{\mathrm{d}}=D_{\ell} \cup \cdots \cup D_{h(N)}$ for some $1 \leqslant \ell \leqslant h(N)$, and remove an element $k$ from it which is maximal in this set (if not, one cannot remove it since $N_{\ell}^{\mathrm{d}} \backslash\{k\}$ would not be a downset). Note that $k \in D_{\ell}$. Then there exists an element $j$ such that $k \prec \cdot j$. Therefore the ray $1^{k}-1^{j}$ is not deleted by $N_{\ell}^{\mathrm{d}} \backslash\{k\}$. Since $k \in D_{\ell}$, it follows that $k \notin N_{\ell+1}^{\mathrm{d}}$, so that ray $1^{k}-1^{j}$ remains. 
A third remarkable collection is the one proposed by Grabisch and Xie (2011), and is defined as follows: $\mathcal{N}^{\mathrm{GX}}=\left\{L_{0}, L_{0} \cup L_{1}, \ldots, L_{0} \cup \cdots \cup L_{h(N)-1}\right\}$. It is a short nested collection, and as observed by Grabisch (2011), there is no short nested collection such that each set includes a set of $\mathcal{N}^{\mathrm{GX}}$. Observe that the downwards collection can be seen as a "dual" of $\mathcal{N}^{\mathrm{GX}}$, since $\mathcal{N}^{\mathrm{d}}=\left\{D_{1} \cup \cdots \cup D_{h(N)}, \ldots, D_{h(N)-1} \cup D_{h(N)}, D_{h(N)}\right\}$.

Example 1. Consider the poset $(N, \leqslant)$ of 9 elements depicted below.

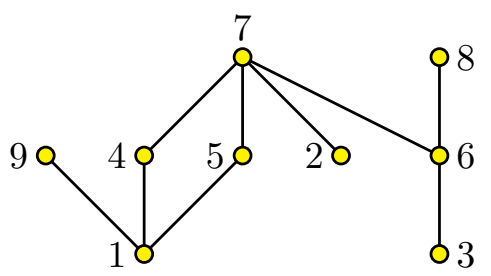

We have $L_{0}=\{1,2,3\}, L_{1}=\{4,5,6,9\}, L_{2}=\{7,8\}$, and $D_{0}=\{7,8,9\}, D_{1}=\{2,4,5,6\}$, $D_{2}=\{1,3\}$. The upwards collection is $\{123,13456\}$, the downwards collection is $\{13,123456\}$, and the Grabisch-Xie collection is $\{123,1234569\}$ (where it is understood that 123 is a shorthand for $\{1,2,3\}$, etc.).

\subsection{Nested closure of a normal collection}

Let $\mathcal{N}$ be a normal collection and let $q$ denote its cardinality. An ordering of $\mathcal{N}$ is a bijection $\sigma: \mathcal{N} \rightarrow\{1, \ldots, q\}$. For any ordering $\sigma$ define its corresponding nested closure by $\overline{\mathcal{N}}_{\sigma}=\left\{\bigcup_{i=1}^{k} \sigma^{-1}(i) \mid k=1, \ldots, q\right\}$. We say that $\sigma$ is a feasible ordering of $\mathcal{N}$ if

$$
\sigma^{-1}(k) \backslash\left(\sigma^{-1}(k-1) \cup \cdots \cup \sigma^{-1}(1)\right) \text { is an antichain of }(N, \preceq) \text { for all } k=1, \ldots, q \text {, }
$$

where $\sigma^{-1}(0)=\emptyset$.

Example 2. (Example 1 cont.) Consider the normal collection $\mathcal{N}=\{136,134,125\}$. Then $\mathcal{N}$ has no feasible ordering because none of its elements is an antichain.

Theorem 1. Let $\mathcal{N}$ be a normal collection and $\sigma$ be an ordering of $\mathcal{N}$. Then the nested closure $\overline{\mathcal{N}}_{\sigma}$ is normal if and only if $\sigma$ is a feasible ordering of $\mathcal{N}$.

Proof. Consider a normal collection $\mathcal{N}$, an ordering $\sigma$ of $\mathcal{N}$, and denote by $\overline{\mathcal{N}}_{\sigma}=$ $\left\{\bar{N}_{1}, \ldots, \bar{N}_{q}\right\}$ its nested closure, i.e., $\bar{N}_{k}=\bigcup_{i=1}^{k} \sigma^{-1}(i)$.

Suppose that $\overline{\mathcal{N}}_{\sigma}$ is not normal. Then there exists a ray $1^{i}-1^{j}$ with $i \prec \cdot j$ which is not deleted by $\overline{\mathcal{N}}_{\sigma}$, i.e., any set in $\overline{\mathcal{N}}_{\sigma}$ either contains both $i$ and $j$ or none of them. Since $\overline{\mathcal{N}}_{\sigma}$ is nested, there exists some $k \in\{1, \ldots, q\}$ such that $\bar{N}_{1}, \ldots, \bar{N}_{k}$ contain neither $i$ nor $j$, and $\bar{N}_{k+1}, \ldots, \bar{N}_{q}$ contain both $i$ and $j$. Therefore, in $\mathcal{N}, \sigma^{-1}(1), \ldots, \sigma^{-1}(k)$ contain neither $i$ nor $j$, while $\sigma^{-1}(k+1)$ contains them both. Since $i \prec \cdot j$, this contradicts (3), hence $\sigma$ is not a feasible ordering.

Conversely, suppose that $\sigma$ is not feasible for $\mathcal{N}$. Then there exist $i, j \in N$ with $i \prec j$, and $k \in\{1, \ldots, q\}$ such that $\sigma^{-1}(k)$ contains both $i, j$, while the sets $\sigma^{-1}(k-1), \ldots, \sigma^{-1}(1)$ contain none of them. Then, $\bar{N}_{k} \backslash \bar{N}_{k-1}$ is not an antichain, and by Lemma 3 , we conclude that $\overline{\mathcal{N}}_{\sigma}$ is not normal. 
Proposition 3. For the upwards collection the ordering $\sigma$ defined by $\sigma\left(N_{k}^{\mathrm{u}}\right)=k$, for $k=1, \ldots, h(N)$, is feasible.

Proof. Noting that $\min \left(N \backslash\left(L_{0} \cup \cdots \cup L_{k-2}\right)\right)=L_{k-1}$, we see that $N_{k}^{\mathrm{u}} \subseteq L_{0} \cup \cdots \cup L_{k-1}$. Therefore, elements in $N_{k}^{\mathrm{u}} \cap L_{k-1}$ do not belong to $N_{1}^{\mathrm{u}} \cup \cdots \cup N_{k-1}^{\mathrm{u}}$. We claim that if $x \in$ $N_{k}^{\mathrm{u}} \cap\left(L_{0} \cup \cdots \cup L_{k-2}\right)$, then it belongs to $N_{1}^{\mathrm{u}} \cup \cdots \cup N_{k-1}^{\mathrm{u}}$. Therefore, $N_{k}^{\mathrm{u}} \backslash\left(N_{1}^{\mathrm{u}} \cup \cdots \cup N_{k-1}^{\mathrm{u}}\right)$ is an antichain, since it is a subset of $L_{k-1}$. This being true for $k=1, \ldots h(N)$, it follows that $\sigma$ is a feasible ordering.

Proof of the claim: Suppose w.l.o.g. that $x \in L_{j}$ for some $j \in\{0, \ldots, k-2\}$. Clearly, $x \notin N_{1}^{\mathrm{u}} \cup \cdots \cup N_{j}^{\mathrm{u}}$. Suppose that $x \notin N_{j+1}^{\mathrm{u}}$. Since $N_{j+1}^{\mathrm{u}}=\downarrow \min \left(\left(L_{j} \cup \cdots \cup L_{h(N)}\right)^{\circ}\right)$, it follows that $x$ is disconnected in $L_{j} \cup \cdots \cup L_{h(N)}$, i.e., there is no $y \in L_{j} \cup \cdots \cup L_{h(N)}$ such that $x \prec y$. But $x \notin L_{k-1}$ and $x \in N_{k}^{\mathrm{u}}$ (which is a downset) imply that it must exist some $y \in L_{k-1}$ such that $x \prec y$, a contradiction. Therefore $x \in N_{j+1}^{\mathrm{u}}$.

It follows by Theorem 1 that the nested closure of $\mathcal{N}^{\mathrm{u}}$ is a normal collection, called by Grabisch (2011) the Weber collection $\mathcal{N}^{\mathrm{W}}=\left\{N_{1}^{\mathrm{W}}, \ldots, N_{h(N)}^{\mathrm{W}}\right\}$, and we have

$$
\begin{aligned}
N_{1}^{\mathrm{W}} & =N_{1}^{\mathrm{u}} \\
N_{2}^{\mathrm{W}} & =N_{1}^{\mathrm{u}} \cup N_{2}^{\mathrm{u}} \\
\vdots & =\vdots \\
N_{h(N)}^{\mathrm{W}} & =N_{1}^{\mathrm{u}} \cup \cdots \cup N_{h(N)}^{\mathrm{u}} .
\end{aligned}
$$

The Weber collection is short, therefore minimal, but not thin in general, as shown by the next result and Example 3.

Proposition 4. Consider the downwards and the Weber collections. It holds

$$
N_{h(N)}^{\mathrm{d}} \subseteq N_{1}^{\mathrm{W}}, \quad N_{h(N)-1}^{\mathrm{d}} \subseteq N_{2}^{\mathrm{W}}, \ldots, \quad N_{1}^{\mathrm{d}} \subseteq N_{h(N)}^{\mathrm{W}} .
$$

Proof. Recall that the Weber collection is $\left\{N_{1}^{\mathrm{u}}, N_{1}^{\mathrm{u}} \cup N_{2}^{\mathrm{u}}, \ldots, N_{1}^{\mathrm{u}} \cup N_{2}^{\mathrm{u}} \cup \cdots \cup N_{h(N)}^{\mathrm{u}}\right\}$. We will prove (4) by induction. We first prove that $N_{h(N)}^{\mathrm{d}} \subseteq N_{1}^{\mathrm{u}}$. Take $i \in N_{h(N)}^{\mathrm{d}}$. Then $i$ is covered by some $j$, and does not cover any element in $N$ by construction. Therefore $i$ is minimal in $N$ and is not disconnected, hence it belongs to $N_{1}^{\mathrm{u}}$.

Induction hypothesis: $N_{h(N)-l+1}^{\mathrm{d}} \subseteq N_{1}^{\mathrm{u}} \cup \cdots \cup N_{l}^{\mathrm{u}}$ for $l=1, \ldots, k<h(N)$. Let us prove that the property still holds for $l=k+1$. Take any $i \in N_{h(N)-k}^{\mathrm{d}} \backslash N_{h(N)-k+1}^{\mathrm{d}}=D_{h(N)-k}$ and note that it is not a disconnected element in $(N, \preceq)$. Then either $i$ covers some $j \in N_{h(N)-k+1}^{\mathrm{d}}$, or $i$ is incomparable with these elements. If $i$ covers some $j$ in $N_{h(N)-k+1}^{\mathrm{d}}$, since by induction hypothesis, all these $j$ 's are members of $N_{1}^{\mathrm{u}} \cup \cdots \cup N_{k}^{\mathrm{u}}$, either $i$ belongs to $N_{1}^{\mathrm{u}} \cup \cdots \cup N_{k}^{\mathrm{u}}$, or to $\min \left(\left(N \backslash\left(N_{1}^{\mathrm{u}} \cup \cdots \cup N_{k}^{\mathrm{u}}\right)\right)^{\circ}\right)$, in which case $i \in N_{k+1}^{\mathrm{u}}$. On the other hand, if $i$ is incomparable with elements of $N_{h(N)-k+1}^{\mathrm{d}}$, it does not cover any element in $N$ (otherwise this element would belong to $N_{h(N)-k+1}^{\mathrm{d}}$, and $i$ would be comparable with an element of $N_{h(N)-k+1}^{\mathrm{d}}$, a contradiction), which implies that it is minimal in $N$, and therefore belongs to $N_{1}^{\mathrm{u}}$.

Example 3. (Example 1 continued) The Weber collection is 123,123456, hence in the above proposition there are cases where strict inclusion occurs for some set. 
Take any nested normal collection $\mathcal{N}=\left\{N_{1}, \ldots, N_{q}\right\}, N_{1} \subset \cdots \subset N_{q}$, and define its (unnested) opening by

$$
\mathcal{N}^{\circ}=\left\{N_{1}, \downarrow\left(N_{2} \backslash N_{1}\right), \ldots, \downarrow\left(N_{q} \backslash\left(N_{1} \cup \cdots \cup N_{q-1}\right)\right)\right\} .
$$

Proposition 5. If $\mathcal{N}$ is a nested normal collection, then its opening $\mathcal{N}^{\circ}$ is normal and there exists a feasible ordering of $\mathcal{N}^{\circ}$ whose corresponding nested closure is $\mathcal{N}$.

Proof. Suppose $\mathcal{N}^{\circ}$ is not normal. Then there exists a ray $1^{i}-1^{j}$, with $i \prec j$, which is not deleted by $\mathcal{N}^{\circ}$, i.e., all sets in $\mathcal{N}^{\circ}$ either contain both $i, j$ or none of them. Hence, the same property holds for the sets in $\mathcal{N}$, which implies that $\mathcal{N}$ is not normal, a contradiction.

Put $\mathcal{N}^{\circ}=\left\{N_{1}^{\circ}, \ldots, N_{q}^{\circ}\right\}$. Since $\mathcal{N}^{\circ}$ and $\mathcal{N}$ are normal, and $\overline{\mathcal{N}}_{\sigma}^{\circ}=\mathcal{N}$ with $\sigma\left(N_{k}^{\circ}\right)=k$ for $k=1, \ldots, q$, by Theorem 1 , it follows that $\sigma$ is feasible.

Corollary 1. Every nested normal collection arises as the closure of a normal collection for which there is a feasible ordering, or is itself an open collection (i.e., invariant under the opening).

\section{Balancedness conditions}

Let $\mathcal{N}$ be a normal collection and $(\mathcal{F}, v)$ a game. Whether $\mathcal{C}_{\mathcal{N}}(v)$ is empty or not depends on both the normal collection and the game. It may happen that $\mathcal{C}_{\mathcal{N}}(v)$ is empty while $\mathcal{C}(v)$ is not. If $\mathcal{C}_{\mathcal{N}}(v)$ is nonempty, then it is a bounded face of $\mathcal{C}(v)$.

The following result has been shown by Grabisch (2011).

Proposition 6. Suppose that $(\mathcal{F}, v)$ is a convex game. Then for any normal nested collection $\mathcal{N}$, the restricted core $\mathcal{C}_{\mathcal{N}}(v)$ is nonempty.

A direct application of the Farkas Lemma leads to the following.

Proposition 7. Let $\mathcal{N}$ be a normal collection. $\mathcal{C}_{\mathcal{N}}(v) \neq \emptyset$ if and only if for all vectors $y \in \mathbb{R}^{\mathcal{F} \backslash\{\emptyset\}}$ satisfying

$$
\begin{aligned}
& \sum_{S \ni i, S \in \mathcal{F}} y_{S}=0, \quad i \in N \\
& y_{S} \geqslant 0, \quad S \in \mathcal{F} \backslash\{\mathcal{N} \cup N\},
\end{aligned}
$$

it holds

$$
\sum_{S \in \mathcal{F} \backslash\{\emptyset\}} y_{S} v(S) \leqslant 0 .
$$

Another form can be obtained proceeding as in the classical Bondareva-Shapley theorem (Bondareva, 1963; Shapley, 1971). Considering a normal collection $\mathcal{N}$, we say that a collection $\mathcal{B} \subseteq \mathcal{F}$ is $\mathcal{N}$-balanced if there exists $y_{S}>0, S \in \mathcal{B}$, such that $\sum_{S \in \mathcal{B}} y_{S} 1^{S}=\sum_{S \in \mathcal{N} \cup N} 1^{S}$. We call $\left\{y_{S}\right\}_{S \in \mathcal{B}}$ a system of $\mathcal{N}$-balancing weights.

Theorem 2. Let $\mathcal{N}$ be a normal collection. $\mathcal{C}_{\mathcal{N}}(v) \neq \emptyset$ if and only if for every $\mathcal{N}$-balanced collection $\mathcal{B}$ with $\mathcal{N}$-balancing weights $\left\{y_{S}\right\}_{S \in \mathcal{B}}$, it holds

$$
\sum_{S \in \mathcal{B}} y_{S} v(S) \leqslant \sum_{S \in \mathcal{N} \cup N} v(S)
$$


Proof. We consider the following linear program with $x \in \mathbb{R}^{n}$ :

$$
\begin{aligned}
\min & =\sum_{S \in \mathcal{N} \cup N} x(S) \\
\text { s.t. } x(S) & \geqslant v(S), \quad S \in \mathcal{F}
\end{aligned}
$$

The optimal value $z^{*}$ of $z$ is $\sum_{S \in \mathcal{N} \cup N} v(S)$ if and only if $\mathcal{C}_{\mathcal{N}}(v) \neq \emptyset$. The dual problem reads

$$
\begin{aligned}
& \max \quad w=\sum_{S \in \mathcal{F}} y_{S} v(S) \\
& \text { s.t } \quad \sum_{S \ni i, S \in \mathcal{F}} y_{S}=\sum_{S \ni i, S \in \mathcal{N} \cup N} y_{S}, \quad i \in N \\
& y_{S} \geqslant 0, \quad S \in \mathcal{F} \text {. }
\end{aligned}
$$

By the duality theorem, $w^{*}=z^{*}$, which implies that any feasible solution satisfies $\sum_{S \in \mathcal{F}} y_{S} v(S) \leqslant \sum_{S \in \mathcal{N} \cup N} v(S)$.

Note that this generalizes a result by Grabisch and Xie (2011). As it can be observed, the proof follows the classical argument, where in the right members of (in)equalities, $N$ is formally replaced by $\mathcal{N} \cup N$. Therefore, a strong form of the theorem can be obtained as well, which we give without proof.

We say that a collection $\mathcal{B} \subseteq \mathcal{F}$ is minimal $\mathcal{N}$-balanced if $\mathcal{B}$ is $\mathcal{N}$-balanced and no proper subcollection is. Similarly to the classical case, we obtain that a minimal $\mathcal{N}$ balanced collection has a unique system of $\mathcal{N}$-balancing weights, and we get the following result.

Theorem 3. Let $\mathcal{N}$ be a normal collection. $\mathcal{C}_{\mathcal{N}}(v) \neq \emptyset$ if and only if (5) holds for any minimal $\mathcal{N}$-balanced collection $\mathcal{B}$, where $\left\{y_{S}\right\}_{S \in \mathcal{B}}$ is the unique system of $\mathcal{N}$-balancing weights for $\mathcal{B}$.

Convexity is not a necessary condition for balancedness. Indeed, Grabisch and Sudhölter (to appear, Lemma 3.2) have shown that if $(N, \preceq$ ) is connected (that is, for any $i, j \in N$, there is a sequence of elements $i=i_{1}, i_{2}, \ldots, i_{k}=j$ such that $i_{\ell}$ and $i_{\ell+1}$ are comparable, for $\ell=1, \ldots, k-1)$, then the core $\mathcal{C}(\mathcal{F}, v)$ is nonempty for any game $v$, in particular for nonconvex games. Since $\mathcal{C}(\mathcal{F}, v)$ contains no line, the bounded core too is nonempty, implying that there exists a nonempty restricted core for any game $v$.

\section{The case of convex games}

By Proposition 6, we know that for convex games, nested normal collections play a central rôle. The next result proves that moreover, one only needs to consider nested normal collections, and the main result of this section (Theorem 5) will show the converse of Proposition 6 for strictly convex games.

Proposition 8. For any normal collection $\mathcal{N}$, there exists a nested normal collection $\mathcal{N}^{\prime}$ such that, for any convex game $v$,

$$
\mathcal{C}_{\mathcal{N}}(v) \subseteq \mathcal{C}_{\mathcal{N}^{\prime}}(v)
$$

The proof is based on the following technical lemma. For any collection $\emptyset \neq \mathcal{G} \subseteq \mathcal{F}$ we define

$$
F(\mathcal{G})=\left\{\left(\mathcal{G} \backslash\left\{T, T^{\prime}\right\}\right) \cup\left\{T \cap T^{\prime}, T \cup T^{\prime}\right\} \mid T, T^{\prime} \in \mathcal{G}\right\} .
$$

Note that any element of $F(\mathcal{G})$ is nonempty and does not possess a larger cardinality than $\mathcal{G}$ and that $\mathcal{G} \in F(\mathcal{G})$. 
Lemma 6. With $g=|\mathcal{G}|$, the $\frac{g(g-1)}{2}$-fold composition of $F$ applied to $\mathcal{G}$, i.e., $F^{\frac{g(g-1)}{2}}(\mathcal{G})$, contains a nested collection.

Proof. We proceed by induction on $g$. If $g=1$, then $\mathcal{G}$ is already nested. Assume that the lemma is valid for any $g<k$ for some $k>1$. Now, if $g=k$, then let $\mathcal{G}=\left\{T_{1}, \ldots, T_{g}\right\}$, define $T_{1}^{\prime}=\bigcup_{j=1}^{g} T_{j}, T_{k}^{\prime}=T_{k} \cap \bigcup_{j=1}^{k-1} T_{k}$ for $k=2, \ldots, g$ and let $\mathcal{G}^{\prime}=\left\{T_{1}^{\prime}, \ldots, T_{g}^{\prime}\right\}$. Note that $\mathcal{G}^{\prime} \in F^{g-1}(\mathcal{G})$ and that $2 \leqslant g^{\prime}=\left|\mathcal{G}^{\prime}\right| \leqslant g$. By the inductive hypothesis, $F^{\frac{\left(g^{\prime}-1\right)\left(g^{\prime}-2\right)}{2}}\left(\mathcal{G}^{\prime} \backslash\left\{T_{1}^{\prime}\right\}\right)$ contains a nested collection $\mathcal{G}^{\prime \prime}$. By construction all elements of $\mathcal{G}^{\prime \prime}$ are contained in $T_{1}^{\prime}$. Hence, $\mathcal{G}^{\prime \prime} \cup\left\{T_{1}^{\prime}\right\}$ is a nested collection in $F^{g-1+\frac{\left(g^{\prime}-1\right)\left(g^{\prime}-2\right)}{2}}(\mathcal{G}) \subseteq$ $F^{\frac{g(g-1)}{2}}(\mathcal{G})$.

Proof. (of Proposition 8) We may assume that $\mathcal{N} \neq \emptyset$. Let $T, T^{\prime} \in \mathcal{N}$ and $v$ be a convex game. In view of Lemma 6 it suffices to show that $\mathcal{N}^{\prime}=\left(\mathcal{N} \backslash\left\{T, T^{\prime}\right\}\right) \cup\left\{T \cup T^{\prime}, T \cap T^{\prime}\right\}$ is (a) normal and (b) $\mathcal{C}_{\mathcal{N}}(v) \subseteq \mathcal{C}_{\mathcal{N}^{\prime}}(v)$. In view of Lemma 2 and by interchanging the roles of $T$ and $T^{\prime}$ if necessary, in order to show (a) it suffices to prove that, for any $i, j \in N$ such that $i \in T \not \supset j$ and $i \prec \cdot j$ either $j \notin T \cup T^{\prime}$ or $i \in T \cap T^{\prime}$. Now, if $j \in T \cup T^{\prime}$, i.e., $j \in T^{\prime}$, then $i \in T^{\prime}$ because $T^{\prime}$ is a downset. Hence, $i \in T \cap T^{\prime}$ in this case. In order to show (b) let $x \in \mathcal{C}_{\mathcal{N}}(v)$. In order to show that $x \in \mathcal{C}_{\mathcal{N}^{\prime}}(v)$ it suffices to show that $x\left(T \cup T^{\prime}\right)=v\left(T \cup T^{\prime}\right)$ and $x\left(T \cap T^{\prime}\right)=v\left(T \cap T^{\prime}\right)$. As the game is convex,

$$
\begin{gathered}
v\left(T \cup T^{\prime}\right)+v\left(T \cap T^{\prime}\right) \leqslant x\left(T \cup T^{\prime}\right)+x\left(T \cap T^{\prime}\right) \\
=x(T)+x\left(T^{\prime}\right)=v(T)+v\left(T^{\prime}\right) \leqslant v\left(T \cup T^{\prime}\right)+v\left(T \cap T^{\prime}\right)
\end{gathered}
$$

so that the desired equalities follow immediately.

We recall the notion of marginal vector and restricted marginal vector introduced by Grabisch (2011). We consider the set of maximal chains in $\mathcal{F}$. This set is in a one-toone correspondence with the set $\mathcal{L}(\preceq)$ of linear extensions of $(N, \preceq)$, i.e., to any maximal chain $C=\left\{\emptyset, S_{1}, S_{2}, \ldots, S_{n}\right\}, \emptyset \subset S_{1} \subset \cdots \subset S_{n}=N$, corresponds a unique permutation $\pi$ on $N$ with $S_{i}:=\{\pi(1), \ldots, \pi(i)\}, i=1, \ldots, n$, and vice versa. The linear extension is given by the sequence $\pi(1), \pi(2), \ldots, \pi(n)$. Considering a game $(\mathcal{F}, v)$, the marginal vector $a^{\pi}(v) \in \mathbb{R}^{N}$ associated to the linear extension $\pi$ (equivalently, $a^{C}(v)$ associated to the maximal chain $C$ ) is the payoff vector defined by

$$
a_{\pi(i)}^{\pi}(v):=v\left(S_{i}\right)-v\left(S_{i-1}\right)=v(\{\pi(1), \ldots, \pi(i)\})-v(\{\pi(1), \ldots, \pi(i-1)\}), \quad i \in N .
$$

Consider a nested collection $\mathcal{G}$ (not necessarily normal). A restricted maximal chain w.r.t. $\mathcal{G}$ is a maximal chain (from $\emptyset$ to $N$ ) in $\mathcal{F}$ containing $\mathcal{G}$. Associated linear extensions are called restricted linear extensions, and the set of restricted linear extensions w.r.t. $\mathcal{G}$ is denoted by $\mathcal{L}_{\mathcal{G}}(\preceq)$. A restricted marginal vector is a marginal vector whose underlying maximal chain is restricted.

The following result is noteworthy and extends the classical result of Shapley (1971) and Ichiishi $(1981)^{1}$.

\footnotetext{
${ }^{1}$ This result is in fact already known. It has been proved for acyclic permission structures by Derks and Gilles (1995), while it is known from Algaba et al. (2004) that these set systems are equivalent to set systems of the form $\mathcal{O}(N)$. Also, Grabisch and Xie (2008) proved it in an unpublished paper. The "only if" part is known from Fujishige and Tomizawa (1983) (also cited in (Fujishige, 2005, Theorem 3.22)). We provide a simpler proof of this result, thereby also making the current paper more self-contained.
} 
Theorem 4. A game $(\mathcal{F}, v)$ is convex if and only if $a^{\pi}(v) \in \mathcal{C}(v)$ for every $\pi \in \mathcal{L}(\preceq)$.

Proof. Necessity: Assume that $v$ is convex, let $S \in \mathcal{F} \backslash\{\emptyset\}$, and $\pi \in \mathcal{L}(\preceq)$. We have to show that

$$
\sum_{i \in S} a_{i}^{\pi}(v) \geqslant v(S)
$$

Let $i_{1}, \ldots, i_{s} \in S, s=|S|$, be chosen so that $\pi^{-1}\left(i_{1}\right)<\cdots<\pi^{-1}\left(i_{s}\right)$. Then $T_{k}=$ $\left\{i_{1}, \ldots, i_{k}\right\}=S_{\pi^{-1}\left(i_{k}\right)} \cap S \in \mathcal{F}$ for any $k=1, \ldots, s$, using the above notation. By $(2)$,

$$
v\left(S_{\pi^{-1}\left(i_{k}\right)}\right)-v\left(S_{\pi^{-1}\left(i_{k}\right)} \backslash i_{k}\right) \geqslant v\left(T_{k}\right)-v\left(T_{k-1}\right) \text { for all } k=1, \ldots, s,
$$

where $T_{0}=\emptyset$. Summing up all these inequalities yields (6).

Sufficiency: Let $v$ be a game and assume that $a^{\pi}(v) \in \mathcal{C}(v)$ for all $\pi \in \mathcal{L}(\preceq)$. Let $S, T \in \mathcal{F}$ so that $S \backslash T \neq \emptyset \neq T \backslash S$. Let $S \cap T=\left\{i_{1}, \ldots, i_{r}\right\}, T \backslash S=\left\{i_{r+1}, \ldots, i_{t}\right\}$, $S \backslash T=\left\{i_{t+1}, \ldots, i_{q}\right\}$, and $N \backslash(S \cup T)=\left\{i_{q+1}, \ldots, i_{n}\right\}$ such that, for any $j \in N$, $\left\{i_{1}, \ldots, i_{j}\right\} \in \mathcal{F}$. Then the permutation $\pi$ defined by $\pi(j)=i_{j}$ for any $j \in N$ is a linear extension. Hence

$$
\begin{aligned}
v(S) \leqslant & \sum_{i \in S} a_{i}^{\pi}(v)=\sum_{i \in S}\left(v\left(S_{\pi^{-1}(i)}\right)-v\left(S_{\pi^{-1}(i)} \backslash i\right)\right. \\
= & \sum_{j=1}^{r}\left(v\left(\left\{i_{1}, \ldots, i_{j}\right\}\right)-v\left(\left\{i_{1}, \ldots, i_{j-1}\right\}\right)\right)+\sum_{j=t+1}^{q}\left(v\left(T \cup\left\{i_{t+1}, \ldots, i_{j}\right\}\right)\right. \\
& \left.-v\left(T \cup\left\{i_{t+1}, \ldots, i_{j-1}\right\}\right)\right) \\
= & v(S \cap T)+v(S \cup T)-v(T),
\end{aligned}
$$

so that the proof is complete.

In Grabisch (2011, Theorems 4 and 5), it is proved that for any nested normal collection $\mathcal{N}$, the set of restricted marginal vectors is the set of extreme points of $\mathcal{C}_{\mathcal{N}}(v)$ if $v$ is convex.

Proposition 9. For any nested normal collection $\mathcal{N}$ of $\mathcal{F}$ and any convex game $v$, $\left\{a^{\pi}(v) \mid \pi \in \mathcal{L}_{\mathcal{N}}(\preceq)\right\}$ is the set of extreme points of $\mathcal{C}_{\mathcal{N}}(v)$.

An inspection of the proof shows that the result extends to any nested collection, not necessarily normal.

We are now in position to show the main result of this section. Let $\mathcal{M N \mathcal { N C }}(\mathcal{F})$ denote the set of minimal nested normal collections of $\mathcal{F}$.

Theorem 5. (i) For any convex game $v$ and any nested normal collection $\mathcal{N}$ of $\mathcal{F}$, $\mathcal{C}_{\mathcal{N}}(v) \neq \emptyset$. Moreover, if $v$ is strictly convex, then $\operatorname{dim} \mathcal{C}_{\mathcal{N}}(v)=n-|\mathcal{N}|-1$.

(ii) For any convex game $v$,

$$
\mathcal{C}^{b}(v)=\bigcup_{\mathcal{N} \in \mathcal{M N \mathcal { N C }}(\mathcal{F})} \mathcal{C}_{\mathcal{N}}(v)
$$

Moreover, no term in the union is redundant if $v$ is strictly convex. 
(iii) Let $\mathcal{N}$ be a normal collection of $\mathcal{F}$. If $v$ is strictly convex, then $\mathcal{C}_{\mathcal{N}}(v) \neq \emptyset$ if and only if $\mathcal{N}$ is nested.

Proof. (i) The first assertion is Proposition 6. By Proposition 9, for any $\pi \in \mathcal{L}_{\mathcal{N}}(\preceq)$, $a^{\pi}(v) \in \mathcal{C}_{\mathcal{N}}(v)$. Let $x=\frac{1}{\left|\mathcal{L}_{\mathcal{N}}(\preceq)\right|} \sum_{\pi \in \mathcal{L}_{\mathcal{N}}(\preceq)} a^{\pi}(v)$. If $v$ is strictly convex, then in order to show the equation it suffices to prove that $x(S)>v(S)$ for all $S \in \mathcal{F} \backslash \mathcal{N} \cup\{\emptyset, N\}$. Let $\mathcal{N} \cup\{\emptyset, N\}=\left\{T_{0}, \ldots, T_{r}\right\}$, where $\emptyset=T_{0} \varsubsetneqq T_{1} \varsubsetneqq \cdots \varsubsetneqq T_{r}=N$. Suppose there exists $j \in\{1, \ldots, r-1\}$ such $T_{j} \backslash S \neq \emptyset \neq S \backslash T_{j}$, then

$v(S)+v\left(T_{j}\right)<v\left(S \cap T_{j}\right)+v\left(S \cup T_{j}\right) \leqslant x\left(S \cap T_{j}\right)+x\left(S \cup T_{j}\right)=x(S)+x\left(T_{j}\right)=x(S)+v\left(T_{j}\right)$

by strict convexity and because $x \in \mathcal{C}(v)$. Otherwise there exists $\ell \in\{0, \ldots, r-1\}$ such that $T_{\ell} \varsubsetneqq S \varsubsetneqq T_{\ell+1}$. Let $S^{\prime}=T_{\ell} \cup\left(T_{\ell+1} \backslash S\right)$. Note that since $T_{\ell+1} \backslash T_{\ell}$ is an antichain by Lemma $3, S^{\prime} \in \mathcal{F}$. Then there exists $\tilde{\pi} \in \mathcal{L}_{\mathcal{N}}(\preceq)$ such that $S^{\prime}=\left\{\widetilde{\pi}(1), \ldots, \widetilde{\pi}\left(\left|S^{\prime}\right|\right)\right\}$, i.e., $\sum_{i \in S^{\prime}} a_{i}^{\widetilde{\pi}}(v)=v\left(S^{\prime}\right)$. By strict convexity we conclude that $\sum_{i \in S} a_{i}^{\tilde{\pi}}>v(S)$. For any $\pi \in \mathcal{L}_{\mathcal{N}}(\preceq), \sum_{i \in S} a_{i}^{\pi}(v) \geq v(S)$, hence $x(S)>v(S)$.

(ii) The equation follows from Propositions 8 and 1, and the fact that minimal normal collections give largest restricted cores. In order to show the final statement, let $v$ be strictly convex and let $x$ be defined as in (i). We have seen that $x(S)=v(S)$ if and only if $S \in \mathcal{N} \cup\{\emptyset, N\}$ so that there is no other minimal normal collection $\mathcal{N}^{\prime}$ with $x \in \mathcal{C}_{\mathcal{N}^{\prime}}(v)$.

(iii) One direction follows from (i). For the other direction let $\mathcal{N}$ be a normal collection that is not nested. Hence, there are $S, T \in \mathcal{N}$ such that $S \backslash T \neq \emptyset \neq T \backslash S$. By strict convexity, $v(S)+v(T)<v(S \cup T)+v(S \cap T)$ so that any $y \in \mathbb{R}^{N}$ with $y(S)=v(S)$ and $y(T)=v(T)$ either satisfies $y(S \cap T)<v(S \cap T)$ or $y(S \cup T)<v(S \cup T)$. We conclude that $\mathcal{C}_{\mathcal{N}}(v)=\emptyset$.

It remains to find all nested minimal normal collections. For this, the following lemma is useful.

Lemma 7. The nested normal collection $\mathcal{N}=\left\{N_{1}, \ldots, N_{q}\right\}, \emptyset \neq N_{1} \varsubsetneqq \cdots \varsubsetneqq N_{q}$, is minimal if and only if

(i) $N_{q} \backslash N_{q-1}$ contains an element that is not maximal (w.r.t. $\preceq$ ), and

(ii) $N_{k} \backslash N_{k-1}$ contains an element $i$ that covers some element $j$ (i.e., $j \prec \cdot i$ ) of $N_{k-1} \backslash N_{k-2}$ for $k=2, \ldots, r$, where $N_{0}=\emptyset$.

Proof. If (i) is not satisfied, then $\mathcal{N} \backslash\left\{N_{q}\right\}$ is still normal, and if (ii) is not satisfied for some $k$, then $\mathcal{N} \backslash\left\{N_{k-1}\right\}$ is still normal. In order to verify the opposite implication assume that $\mathcal{N}$ is not minimal and let $p \in\{1, \ldots, q\}$ such that $\mathcal{N} \backslash\left\{N_{p}\right\}$ is still normal. If $p=q$, then $\mathcal{N}$ violates (i), and if $p<q$, then $\mathcal{N}$ violates (ii) for $k=p+1$.

Every $\pi \in \mathcal{L}(\preceq)$ generates a collection $\mathcal{N}^{\pi} \subseteq \mathcal{F}$ defined as follows: Let $0=t_{0}<t_{1}<$ $\cdots<t_{q}<t_{q+1}=n$ be defined by the requirements that

- $\left\{\pi\left(t_{j}+1\right), \ldots, \pi\left(t_{j+1}\right)\right\}$ is an antichain for all $j=0, \ldots, q$; 
- $\left\{\pi\left(t_{j}+1\right), \ldots, \pi\left(t_{j+1}+1\right)\right\}$ is not an antichain for all $j=0, \ldots, q-1$.

Then define $N_{j}=\left\{\pi(1), \ldots, \pi\left(t_{j}\right)\right\}$ for all $j=1, \ldots, q$ and put $\mathcal{N}^{\pi}=\left\{N_{1}, \ldots, N_{q}\right\}$. As $N_{j} \backslash N_{j-1}$ for $j=1, \ldots, q$ is an antichain (where $N_{0}=\emptyset$ ), $\mathcal{N}^{\pi}$ is a nested normal collection. Moreover, $\mathcal{N}^{\pi}$ is a minimal normal collection by Lemma 7 . Conversely, let $\mathcal{N}=\left\{N_{1}, \ldots, N_{q}\right\}, N_{1} \varsubsetneqq \cdots \varsubsetneqq N_{q}$, be a minimal nested normal collection. Choose $\pi \in \mathcal{L}(\preceq)$ such that

- for all $j=1, \ldots, q, \pi^{-1}\left(N_{j}\right)=\left\{1, \ldots,\left|N_{j}\right|\right\}$;

- $\pi\left(\left|N_{k-1}\right|+1\right)$ covers some element of $N_{k-1} \backslash N_{k-2}$ for all $k=2, \ldots, q$.

By Lemma 7 such $\pi$ exists. By construction $\mathcal{N}=\mathcal{N}^{\pi}$.

We summarize that every linear extension ${ }^{2}$ of $(N, \preceq)$ (i.e., every $\mathcal{F}$-admissible ordering of $N$ ) generates a unique minimal nested normal collection of $\mathcal{F}$ and that minimal nested normal collection is generated by some (not necessarily unique) linear extension of $(N, \preceq$ ).

Example 4. Let $N=\{1,2,3,4\}$ and $\preceq$ be determined by $1 \prec 3$ and $2 \prec 4$. Then minimal nested normal collections are

$$
\mathcal{N}_{1}=\{12\}, \mathcal{N}_{2}=\{1,123\} \text {, and } \mathcal{N}_{3}=\{2,124\} .
$$

Note that $\mathcal{N}_{2}$ and $\mathcal{N}_{3}$, although minimal, are not short. The $\mathcal{F}$-admissible permutations are

$$
\begin{gathered}
\pi_{1}=(1,2,3,4), \pi_{2}=(1,2,4,3), \pi_{3}=(2,1,3,4), \pi_{4}=(2,1,4,3), \pi_{5}=(1,3,2,4), \\
\text { and } \pi_{6}=(2,4,1,3) .
\end{gathered}
$$

The permutations $\pi_{1}, \ldots, \pi_{4}$ generate $\mathcal{N}_{1}, \pi_{5}$ generates $\mathcal{N}_{2}$, and $\pi_{6}$ generates $\mathcal{N}_{3}$. However, for any convex game $v$,

$$
\begin{gathered}
\mathcal{C}_{\mathcal{N}_{1}}=\operatorname{conv}\left(\left\{a^{\pi_{1}}(v), \ldots, a^{\pi_{4}}(v)\right\}\right), \quad \mathcal{C}_{\mathcal{N}_{2}}(v)=\operatorname{conv}\left(\left\{a^{\pi_{1}}(v), a^{\pi_{5}}(v)\right\}\right), \\
\text { and } \mathcal{C}_{\mathcal{N}_{3}}(v)=\operatorname{conv}\left(\left\{a^{\pi_{4}}(v), a^{\pi_{6}}(v)\right\}\right) .
\end{gathered}
$$

Finally, if $v(S)=|S|^{2}$ for $S \in \mathcal{F}$, then $v$ is strictly convex and $\frac{a^{\pi_{5}}(v)+a^{\pi_{6}}(v)}{2}=(3,3,5,5) \notin$ $\mathcal{C}^{b}(v)$ so that the bounded core may be non-convex even for convex games.

The remarkable nested collections $\mathcal{N}^{\mathrm{d}}, \mathcal{N}^{\mathrm{GX}}, \mathcal{N}^{\mathrm{W}}$ presented in Section 3.1 are generated by particular linear extensions among those which satisfy the two above requirements. The $\mathcal{N}^{\mathrm{GX}}$ collection is generated by any such linear extension which in addition satisfies

$$
\begin{aligned}
\pi(1), \ldots, \pi\left(\left|L_{0}\right|\right) & \text { is a permutation on } L_{0} \\
\pi\left(\left|L_{0}\right|+1\right), \ldots, \pi\left(\left|L_{0} \cup L_{1}\right|\right) & \text { is a permutation on } L_{1} \\
& \vdots \\
\pi\left(\left|L_{0} \cup \cdots \cup L_{h(N)-2}\right|+1\right), \ldots, \pi\left(\left|L_{0} \cup \cdots \cup L_{h(N)-1}\right|\right) & \text { is a permutation on } L_{h(N)-1} .
\end{aligned}
$$

\footnotetext{
${ }^{2}$ Linear extensions, also known as topological sorting, can be generated in linear time in the number of linear extensions; see, e.g., Pruesse and Ruskey (1994). However, the problem of counting all linear extensions of a finite partial order is \#P-complete; see Brightwell and Winkler (1991).
} 
The downwards collection $\mathcal{N}^{\mathrm{d}}$ is in turn generated by linear extensions which in addition satisfy

$$
\begin{gathered}
\pi(1), \ldots, \pi\left(\left|D_{h(N)}\right|\right) \text { is a permutation on } D_{h(N)} \\
\pi\left(\left|D_{h(N)}\right|+1\right), \ldots, \pi\left(\left|D_{h(N)-1} \cup D_{h(N)}\right|\right) \text { is a permutation on } D_{h(N)-1} \\
\vdots \\
\pi\left(\left|D_{2} \cup \cdots \cup D_{h(N)}\right|+1\right), \ldots, \pi\left(\left|D_{1} \cup \cdots \cup D_{h(N)}\right|\right) \text { is a permutation on } D_{1} .
\end{gathered}
$$

Finally, the Weber collection arises from any linear extension satisfying in addition

$$
\begin{aligned}
& \pi(1), \ldots, \pi\left(\left|\min \left(N^{\circ}\right)\right|\right) \text { is a permutation on } \min \left(N^{\circ}\right) \\
& \pi\left(\left|\min \left(N^{\circ}\right)\right|+1\right), \ldots, \pi\left(\left|\min \left(N^{\circ}\right) \cup \min \left(\left(N \backslash L_{0}\right)^{\circ}\right)\right|\right) \text { is a permutation on } \\
& \min \left(\left(N \backslash L_{0}\right)^{\circ}\right) \\
& \pi\left(\left|\min \left(N^{\circ}\right) \cup \cdots \cup \min \left(\left(N \backslash\left(L_{0} \cup \cdots \cup L_{q-3}\right)\right)^{\circ}\right)\right|+1\right), \ldots \\
& \ldots, \pi\left(\left|\min \left(N^{\circ}\right) \cup \cdots \cup \min \left(\left(N \backslash\left(L_{0} \cup \cdots \cup L_{q-2}\right)\right)^{\circ}\right)\right|\right) \text { is a permutation on } \\
& \min \left(\left(N \backslash\left(L_{0} \cup \cdots \cup L_{q-2}\right)\right)^{\circ}\right) .
\end{aligned}
$$

\section{References}

E. Algaba, J. M. Bilbao, R. van den Brink, and A. Jiménez-Losada. Cooperative games on antimatroids. Discrete Mathematics, 282:1-15, 2004.

J. M. Bilbao, N. Jiménez, E. Lebrón, and H. Peters. The selectope for games with partial cooperation. Discrete Mathematics, 216:11-27, 2000.

J.M. Bilbao and P.H. Edelman. The Shapley value on convex geometries. Discrete Applied Mathematics, 103:33-40, 2000.

G. Birkhoff. On the combination of subalgebras. Proc. Camb. Phil. Soc., 29:441-464, 1933.

O. Bondareva. Some applications of linear programming to the theory of cooperative games. Problemy Kibernet, 10:119-139, 1963. in Russian.

G. Brightwell and P. Winkler. Counting linear extensions. Order, 8:225-242, 1991.

B. A. Davey and H. A. Priestley. Introduction to Lattices and Orders. Cambridge University Press, 1990.

J. Derks and R. Gilles. Hierarchical organization structures and constraints on coalition formation. Int. J. of Game Theory, 24:147-163, 1995.

U. Faigle and W. Kern. The Shapley value for cooperative games under precedence constraints. Int. J. of Game Theory, 21:249-266, 1992. 
S. Fujishige. Submodular functions and optimization, volume 58 of Annals of Discrete Mathematics. Elsevier, Amsterdam, 2nd edition, 2005.

S. Fujishige and N. Tomizawa. A note on submodular functions on distributive lattices. J. of the Operations Research Society of Japan, 26:309-318, 1983.

M. Grabisch. The core of games on ordered structures and graphs. 4OR, 7:207-238, 2009. DOI: $10.1007 /$ s10288-009-0109-9.

M. Grabisch. Ensuring the boundedness of the core of games with restricted cooperation. Annals of Operations Research, 191:137-154, 2011.

M. Grabisch and P. Sudhölter. The bounded core for games with precedence constraints. Annals of Operations Research, to appear. doi: 10.1007/s10479-012-1228-9.

M. Grabisch and L. J. Xie. The restricted core of games on distributive lattices: how to share benefits in a hierarchy. Mathematical Methods of Operations Research, 73: 189-208, 2011.

M. Grabisch and L. J. Xie. The core of games on distributive lattices: how to share benefits in a hierarchy. Technical Report 2008.77, Centre d'Economie de la Sorbonne, 2008 .

T. Ichiishi. Super-modularity: applications to convex games and to the greedy algorithm for LP. J. Econom. Theory, 25:283-286, 1981.

G. Pruesse and F. Ruskey. Generating linear extensions fast. SIAM J. Comput., 23: 373-386, 1994.

L. S. Shapley. Cores of convex games. Int. J. Game Theory, 1:11-26, 1971.

N. Tomizawa. Theory of hyperspace (XVI) - on the structure of hedrons. Papers of the Technical Group on Circuits and Systems CAS82-172, Inst. of Electronics and Communications Engineers of Japan, 1983. In Japanese. 